\title{
Controversie
}

\section{Controversia BH}

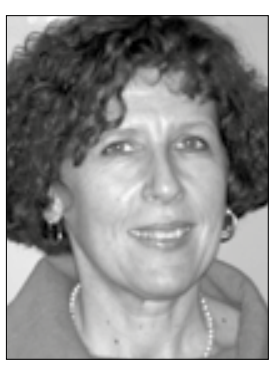

Marisa Pegoraro

\section{Egregio Direttore,}

l'articolo del Dr. Napoli ha un titolo certamente provocatorio, direi anche un po' $^{\prime}$ irritante per i tanti infermieri e medici che da tempo applicano la metodica con la sola volontà di apportare un beneficio al paziente (considerando necessario il maleficio dell'incannulazione). Mi sono apprestata a leggere il contenuto con un certo timore, pensando di trovare chissà quale casistica. Invece, nulla di tutto ciò. Infatti, nell'articolo del Dr. Napoli viene riportata una revisione della bibliografia incompleta rispetto a quella prodotta recentemente da un altro articolo (1) e le omissioni riguardano proprio quegli articoli che non citano infezioni (2-4), quelli che sostengono come una stretta adesione alle procedure e alle prassi sia stata risolutrice (5) e quelli dove si sia attuata una ri-definizione delle competenze assistenziali $(6,7)$ o delle pratiche "ad hoc" per una certa tipologia di pazienti (8, 9). Con questo non si vuole affermare che il $\mathrm{BH}$ non abbia in sé delle criticità, ma che le stesse sono superabili con una "buona pratica clinica". Come tutte le buone pratiche necessita di attenzione, approccio strutturato e momenti di verifica e di riadattamento delle procedure. Questi passaggi non sono "comodi" per chi li deve attuare. Sono certamente, all'inizio, un aumento dell' "attenzione sul lavoro" rispetto alla semplicistica pratica da "un buco e via", e la necessità di un coinvolgimento attivo degli infermieri, anzi, ritengo che vi sia un'enorme componente infermieristica che va sostenuta, stimolata e riconosciuta e, anche, rispettata.

Percepisco una certa malafede in coloro che, pur non avendo provato/sperimentato una nuova prassi, riescono a esprimere giudizi negativi e apparentemente perbenistici. Nel nostro caso, i dati a cui il Dr. Napoli si riferisce, e cioè un'aumentata incidenza di infezione, hanno raramente raggiunto la significatività statistica $(10,11)$ oppure erano imputabili a comportamenti anomali dei pazienti (12). La criticità infettiva ha stimolato chi effettivamente ha applicato la metodica ad apportare una serie di correttivi che si sono rivelati efficaci. Inoltre, il confronto sui dati di esito del $\mathrm{BH}$ rispetto alla puntura a rotazione ha evidenziato una diminuzione di ematomi, stravasi e punture inefficaci $(13,14)$ nel gruppo BH. Non metterei a confronto gli esiti nella gestione dei CVC e $\mathrm{BH}$, ma, piuttosto, buone o cattive pratiche assistenziali: se la potrebbero giustamente prendere a male quei centri che hanno delle eccellenze nella gestione dei CVC e che sono esempio per molti nella gestione delle FAV.

Va sottolineato che il BH esalta la necessità di criteri di competenza assistenziale nell'approccio alla puntura e alla gestione delle fistole, che, oltre a essere confezionate in modo adeguato (al paziente e alla prassi), devono essere gestite con criterio, attenzione e abilità dalle infermiere che se ne occupano. Perché, nella stragrande maggioranza dei centri, sono le/gli infermiere/i che pungono, osservano, scelgono, propongono, valutano e parlano e che educano il paziente. Per questo, la creazione di ruoli di competenza avanzata come il Referente degli Accessi Vascolari (RAV) é un passaggio necessario per i pazienti ed è dovuto alla professione infermieristica, le ragioni del cui agire sono spesso misconosciute da chi paziente non è.

All'approccio cauto, conservativo e ripetitivo dell' "abbiamo sempre fatto così!" io, infermiera di dialisi, in empatia con i miei pazienti, replico che provare con criterio nuove pratiche corrisponda a ricerca: una ricerca della qualità del servizio espresso e percepito, nonché personalizzato. Non ultimo, tutte le nuove pratiche dovrebbero essere uno stimolo alla possibilità di attivare ricerche cliniche applicate, ancora prima di una mera negazione, soprattutto se presenti da tempo in altri contesti. Mi chiedo: se si fosse trattato di un nuovo trattamento diffusivo/convettivo/misto on line, con un filtro di aliena generazione sarebbe stato più facile accettare la ricerca senza troppe remore iniziali? Poiché si tratta, invece, di pratiche cliniche proposte e applicate da infermieri motivati, ecco che, nel nostro contesto, sono passate al setaccio, criticate, vincolate, deformate e defraudate del loro significato principale: diminuire un disagio alla persona, fulcro essenziale del nostro operare nell'ambito sanitario. Chiudo con una domanda per voi lettori, esimi nefrologi, esperti infermieri: se voi foste pazienti di emodialisi, punti tre volte alla settimana con 2 aghi (6 punture) di grosso calibro (e vi invito a provare!) e non sempre dalle mani più caute ed esperte, scegliereste buchi nuovi, dolore nuovo, deformazione del braccio e facili ematomi o, a fronte di un potenziale rischio calcolato ed evitabile, vorreste essere punti in modo da preservare il vostro stato estetico, da contenere il dolore e da limitare ematomi e stravasi e, volendo, in modo da imparare a pungervi da soli? E, comunque, avere la possibilità di scegliere mi pare sia, in se stesso, un evento positivo.

Indirizzo degli Autori:

Dr.ssa Marisa Pegoraro

EDTNA/ERCA Filiale Italiana

c/o DIALISI CAL Corsico

Via Travaglia 5

20094 Corsico (MI)

marisa_pegoraro@fastwebnet.it 


\section{Bibliografia}

1. Pegoraro M. La puntura a occhiello, o buttonhole: quali sono le cose che fanno la differenza. Una revisione della letteratura infermieristica. Wichtig, Milano 2013; Vol. 2/2013.

2. Ward J, Shaw K, Davenport A. Patients' perspectives of constant-site (buttonhole) cannulation for haemodialysis access. Nephron Clin Pract 2010; 116 (2): c123-7.

3. Struthers J, Allan A, Peel RK, Lambie SH. Buttonhole needling of ateriovenous fistulae: a randomized controlled trial. ASAIO J 2010; 56 (4): 319-22.

4. King J. Implementing the buttonhole method using the Biohole peg in a busy dialysis unit: a report of the development of current practice. J Ren Care 2009; 35 (4): 192-200.

5. Labriola L, Jadoul M. Infectious complications following conversion to buttonhole cannulation. Clin Nephrol 2011; 76 (5): 423.

6. Waterhouse D. Vascular access: a role for a renal nurse clinician. EDTNA ERCA J 2002; 28 (2): 64-6, 69.

7. Polkinghorne KR, Seneviratne M, Kerr PG. Effect of a vascular access nurse coordinator to reduce central venous catheter use in incident hemodialysis patients: a quality improvement report. Am J Kidney Dis 2009; 53 (1): 99-106.

8. Nesrallah GE, Cuerden M, Wong JH, Pierratos A. Staphylococcus aureus bacteremia and buttonhole cannulation: long- term safety and efficacy of mupirocin prophylaxis. Clin J Am Soc Nephrol 2010; 5 (6): 1047-53.

9. Van Eps CL, Jones M, Ng T, et al. The impact of extendedhours home hemodialysis and buttonhole cannulation technique on hospitalization rates for septic events related to dialysis access. Hemodial Int 2010; 14 (4): 451-63.

10. Chow J, Rayment G, San Miguel S, Gilbert M. A randomised controlled trial of buttonhole cannulation for the prevention of fistula access complications. J Ren Care 2011; 37 (2): 85-93.

11. MacRae JM, Ahmed SB, Atkar R, Hemmelgarn BR. A randomized trial comparing buttonhole with rope ladder needling in conventional hemodialysis patients. Clin J Am Soc Nephrol 2012; 7 (10): 1632-8.

12. Doss S, Schiller B, Moran J. Buttonhole cannulation--an unexpected outcome. Nephrol Nurs J 2008; 35 (4): 417-9.

13. van Loon MM, Goovaerts T, Kessels AG, et al. Buttonhole needling of haemodialysis arteriovenous fistulae results in less complications and interventions compared to the rope-ladder technique. Nephrol Dial Transplant 2010; 25 (1): 225-30.

14. Verhallen AM, Kooistra MP, van Jaarsveld BC. Cannulating in haemodialysis: rope-ladder or buttonhole technique? Nephrol Dial Transplant 2007; 22 (9): 2601-4.

Accettato: 27 Febbraio 2014 